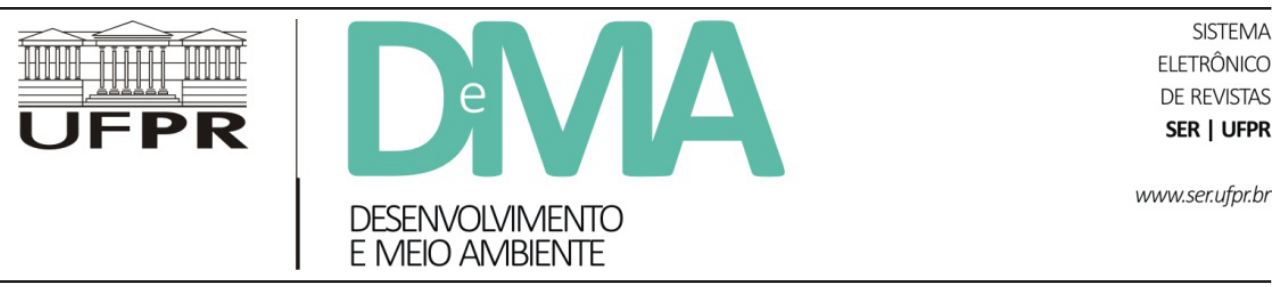

\title{
Difusão de práticas sociais sustentáveis em nichos de inovação social de base: o caso do movimento das ecovilas
}

\section{Diffusion of Sustainable Social Practices in Grassroots Innovation Niches: The Case of the Ecovillage Movement}

\author{
Rebeca ROYSEN ${ }^{*}$, Frédéric MERTENS ${ }^{1}$ \\ ${ }^{1}$ Centro de Desenvolvimento Sustentável, Universidade de Brasília (UnB), Brasília, DF, Brasil. \\ *E-mail de contato: rebecaroysen@gmail.com
}

Ensaio recebido em 10 de maio de 2016, versão final aceita em 3 de novembro de 2016.

RESUMO: Este ensaio busca analisar os processos de inovação e difusão de práticas sociais sustentáveis em nichos de inovação social de base. Para isso, traz elementos da Teoria das Práticas Sociais (TPS), que oferece uma nova perspectiva para o entendimento da difusão de inovações. O texto começa revisando os estudos clássicos sobre difusão de inovações, a perspectiva multinível (PMN) e a TPS para, em seguida, explorar as formas de aplicação da TPS nos estudos sobre difusão de práticas sociais sustentáveis em nichos de inovação social, usando, para tanto, o caso do movimento das ecovilas.

Palavras-chave: difusão de inovações; inovações sociais; práticas sociais; ecovilas; sustentabilidade.

ABSTRACT: This essay analyzes the processes of innovation and diffusion of sustainable social practices in grassroots innovation niches. To do so, it brings elements from social practice theory (SPT), which offers a new perspective on the understanding of the diffusion of innovations. It starts by reviewing the classical studies on diffusion of innovations, the multilevel perspective and the SPT, followed by an exploration of the ways in which SPT can be applied to the studies on diffusion of sustainable social practices in grassroots innovation niches, using as example the case of the ecovillage movement.

Keywords: diffusion of innovations; grassroots innovations; social practice theory; ecovillages; sustainability.

\section{Introdução}

As práticas cotidianas - de consumo, uso/ reuso, reciclagem, mobilidade, refrigeração, higiene, lazer etc. - são comportamentos habituais reproduzidos por milhões de pessoas e que geram impactos no meio ambiente. Diversas correntes têm investigado a mudança comportamental em direção à sustentabilidade a partir de uma ótica individualista, e grande parte desses estudos tem chegado à con- 
clusão de que valores, consciência e preocupação ambientais não se traduzem diretamente em uma mudança de comportamento (Kaiser et al., 1999; Tanner, 1999; Ester et al., 2004; Rodríguez-Barreiro et al., 2013; Roysen, 2015). Isso é chamado, na psicologia social, de lacuna valor-ação (valueaction gap) e pode ser explicado pelo fato de que as práticas cotidianas não surgem unicamente de uma escolha individual e racional, mas estão ligadas às infraestruturas de fornecimento, aos objetos e tecnologias disponíveis, à organização dos espaços, aos significados associados às práticas, às normas sociais, às influências das redes de relações sociais, às competências e hábitos incorporados - enfim, a uma série de elementos que constituem as práticas e que não podem ser localizados no indivíduo isolado (Shove \& Walker, 2010; Spaargaren, 2011; Shove et al., 2015).

A Teoria das Práticas Sociais (TPS) tem buscado superar esse individualismo e criar elos entre os comportamentos individuais, os sistemas tecnológicos e as normas culturais de uma sociedade. Ela traz, portanto, uma nova perspectiva para se pensar a transformação das práticas na direção da sustentabilidade. Alguns estudos sobre inovações têm indicado que um lócus importante de transformação das práticas sociais são os nichos de inovação social de base (grassroots innovation niches). Os nichos de base são grupos de pessoas e organizações da sociedade civil que, motivados por uma ideologia ou pelo desejo de encontrar soluções para as suas necessidades sociais, se organizam, de baixo para cima, para experimentar modelos alternativos e mais sustentáveis de fazer as coisas. Eles criam um espaço seguro para a experimentação, com novas tecnologias, valores e práticas (Seyfang \& Smith, 2007; Seyfang \& Haxeltine, 2012). Os nichos geram, portanto, inovações sociais sustentáveis - aqui entendidas como novas práticas sociais (Cajaiba-
-Santana, 2014) para o desenvolvimento sustentável (Seyfang \& Smith, 2007; Seyfang, 2010).

No Brasil, ainda não existem estudos que investiguem os nichos de inovação social de base ou a transformação das práticas sob a ótica da TPS. Os trabalhos brasileiros sobre difusão de inovações são raros e tendem a se concentrar na difusão de tecnologias e produtos, sem se debruçarem sobre os elementos culturais envolvidos nesses processos (Kimura et al., 2011; Furlanetto \& Santos, 2014; Rodrigues et al., 2014; Moreira \& Vargas, 2015; Moura et al., 2015; Santos \& Gonçalves, 2015). Apesar de diversos estudos internacionais sobre inovações sociais de base terem surgido na última década, ainda sabemos pouco sobre os processos de inovação e as possibilidades de difusão das práticas sociais sustentáveis criadas nesses nichos. O campo da inovação e difusão de práticas sociais - no qual este ensaio se situa - é emergente na literatura internacional e ainda não existe um conjunto estabelecido de conceitos para nortear os estudos empíricos (Shove et al., 2012; Krasny et al., 2015; Shove et al., 2015).

Diversos autores têm ressaltado o potencial da TPS para iluminar esses processos de inovação e difusão das práticas (Shove \& Pantzar, 2005; Shove \& Walker, 2010; Seyfang et al., 2010; Seyfang \& Haxeltine, 2012). O objetivo deste ensaio é, portanto, integrar os conceitos que estão sendo desenvolvidos pela TPS aos estudos sobre difusão de inovações - em especial, a perspectiva multinível (PMN) - para entender os processos de inovação e difusão de práticas sociais sustentáveis nos nichos de inovação social de base.

Usamos como estudo de caso o movimento das ecovilas. Esse movimento é considerado um nicho de inovação social de base (Boyer, 2015; Kunze, 2015a) por ser composto de comunidades locais que experimentam com novas tecnologias 
(na construção das casas e nos sistemas de energia, compostagem, plantio e tratamento de efluentes), mas, principalmente, por se tratar de espaços de intensa experimentação com novas práticas sociais: práticas de compartilhamento de espaços, ferramentas e automóveis, de governança horizontal, de compras coletivas, de alimentação, vestimenta, trabalho e lazer (Kirby, 2003; Christian, 2007; Lockyer, 2008; Veteto \& Lockyer, 2008; Loezer, 2011; Roysen, 2013; Borsos, 2013; Capello, 2013; Kunze, 2015a; Boyer, 2016). Os dados sobre as ecovilas utilizados neste ensaio provêm de referências bibliográficas e da primeira análise de material (entrevistas, grupo focal e observações) recém-coletado em uma pesquisa de campo realizada em uma ecovila no Brasil ${ }^{1}$, durante o mês de julho de 2016.

Este ensaio está dividido em quatro partes. Após esta introdução, buscamos revisar as teorias sobre difusão de inovações: os estudos clássicos, a PMN e a TPS, com foco nas inovações sustentáveis. Em seguida, exploramos como a TPS pode contribuir para a análise da inovação e difusão de práticas sociais sustentáveis, usando como exemplo de nicho de inovação de base o movimento das ecovilas.

\section{Teorias sobre difusão de inovações}

\subsection{Estudos clássicos sobre a difusão de inovações}

Uma das definições mais clássicas de inovação é a de Everett Rogers, segundo o qual uma inovação é "uma ideia, prática ou objeto que é percebido como novo por um indivíduo ou outra unidade de adoção" (Rogers, 1995, p. 11, tradução nossa). Já a difusão, para o mesmo autor, é o "processo pelo qual uma inovação é comunicada, por certos canais, ao longo do tempo, entre os membros de um sistema social. [...] Comunicação é o processo pelo qual os participantes criam e compartilham informações uns com os outros para alcançar um entendimento mútuo" (Rogers, 1995, p. 5, tradução nossa).

Para Rogers, a adoção de uma inovação passa por cinco estágios: (1) primeiro, o indivíduo fica sabendo da existência da inovação. Nessa fase, a percepção seletiva e suas necessidades influenciam as inovações às quais ele estará exposto. Depois de conhecer a inovação, ele (2) forma uma atitude favorável ou desfavorável e (3) toma a decisão de adotar ou rejeitar uma inovação. A fase da implementação é quando o indivíduo (4) coloca a inovação em uso e, por fim, após uma fase de experimentação, ele pode (5) reforçar a escolha feita ou rejeitá-la.

As características das inovações também influenciam a sua taxa de adoção. Tendem a ser mais rapidamente adotadas as inovações que são percebidas pelos indivíduos como tendo as seguintes características: vantagem relativa (em termos econômicos, de prestígio social, conveniência e satisfação); compatibilidade (consistência com os valores existentes, experiências passadas e necessidades dos potenciais adotantes); possibilidade de se experimentar ou de adotar uma inovação de forma gradual; e possibilidade de observação (visibilidade que estimula a discussão e a troca de informação entre pares, amigos e vizinhos).

Essa perspectiva também enfatiza a importância das redes interpessoais na adoção ou rejeição de uma inovação. Diante da incerteza em adotar uma nova tecnologia ou prática, somos influenciados

1 Os nomes da ecovila e de seus membros foram omitidos para preservar as identidades dos sujeitos pesquisados. 
por pessoas parecidas conosco que já a adotaram e que podem nos comunicar a sua experiência. Dessa forma, milhares de estudos têm utilizado a Análise de Redes Sociais (ARS) para entender o processo de difusão (por exemplo: Katz \& Menzel, 1957; Granovetter, 1973; Coleman \& Valente, 1996; Valente et al., 1997; Boulay \& Valente, 1999; Choi et al., 2010).

Quando se fala em sustentabilidade, diversos autores dessa linha têm pesquisado as barreiras e oportunidades na difusão de inovações tecnológicas e práticas sustentáveis, tais como tecnologias e serviços de energias renováveis (Sidiras, 2004; Ozaki, 2011; Hoppe, 2012; Mani \& Dhingra, 2012), tecnologias de economia de água (Schwarz \& Ernst, 2009), práticas alimentares sustentáveis (Mertens et al., 2012) e agricultura orgânica (Läpple \& Rensburg, 2011).

É interessante notar que algumas pesquisas dessa corrente têm identificado barreiras de difusão que se relacionam não com a tecnologia em si nem com a estrutura da rede social dos potenciais adotantes, mas, sim, com a mudança comportamental exigida pela nova tecnologia. Em seu estudo sobre a difusão dos banheiros na Índia, Ramani et al. (2012) demonstram que "sua adoção bem-sucedida exige uma mudança no comportamento individual, nas rotinas diárias e, talvez, mesmo nas normas sociais" (p. 677, tradução nossa). Ao pesquisar a difusão de sistemas produto-serviço, Ceschin (2013) afirma que a sua principal barreira é a mudança cultural necessária para que as pessoas valorizem soluções baseadas no compartilhamento e no acesso em vez de na propriedade.

É muito difícil pensar a produção e o uso de novas tecnologias sem levar em consideração os valores, as normas e as práticas sociais envolvidos nesses processos. No entanto, esses elementos são pouco explorados na perspectiva clássica da difusão de inovações. Apesar da importância dada às redes de relações interpessoais na difusão, os estudos dentro desse referencial tendem a ver o processo de adoção como uma ação essencialmente racional (Camboni \& Napier, 1993; Choi et al., 2010; Läpple \& Rensburg, 2011). Diante dessa lacuna, a PMN vem enfatizar as barreiras sociais e culturais às inovações tecnológicas e a importância dos nichos de inovação para a criação de soluções mais radicais na direção da sustentabilidade (Kemp et al., 1998).

\subsection{Perspectiva multinivel}

A PMN afirma que as transições para a sustentabilidade exigem mudanças em múltiplas dimensões: não apenas nas tecnologias, mas também nos mercados, nas políticas públicas, nas práticas dos usuários e nos significados culturais (Geels, 2010). A PMN distingue três níveis analíticos: os nichos de inovação, os regimes sociotécnicos e a paisagem sociotécnica. As transições seriam mudanças nos regimes, que se dão por meio de processos de interação entre esses três níveis.

Os regimes sociotécnicos referem-se aos sistemas institucionalizados e dominantes de se atender às atuais necessidades societais, incluindo os sistemas de alimentação, comunicação, transportes, saneamento etc. A constituição desses regimes exige investimentos em máquinas e infraestruturas, subsídios e regulações, assim como a adaptação dos estilos de vida, das práticas sociais e dos valores e normas culturais aos sistemas técnicos. Tudo isso cria barreiras sociais, econômicas, tecnológicas e cognitivas às novas tecnologias, os chamados mecanismos de trancamento (lock-in) (Kemp et al., 1998; Geels \& Schot, 2007; Seyfang \& Smith, 2007; Geels, 2010; Smith et al., 2010). Dessa forma, quando surgem inovações nesses regimes, elas 
tendem a ser incrementais ou "normais". Mudanças radicais ou revolucionárias só podem emergir nos chamados "nichos" (Smith et al., 2010).

Nichos são incubadoras, isto é, espaços protegidos em que alternativas radicais podem ser desenvolvidas longe da pressão competitiva prevalecente no regime. Nichos sustentáveis são redes de experimentações com práticas sociotécnicas social e ecologicamente benignas (Smith et al., 2010). Quando a paisagem sociotécnica muda (mudanças demográficas, ambientais, políticas, científicas, econômicas, ideológicas e culturais), ela pode causar pressão para a mudança nos regimes e, assim, abrir janelas de oportunidades para a difusão e adoção das alternativas desenvolvidas nos nichos. Segundo Smith et al. (2010), o sucesso dos nichos depende do envolvimento crescente de atores poderosos, de forma a mobilizar ampla legitimação social. Dessa forma, o potencial transformativo dos nichos é limitado, possibilitado e interpretado pelas estruturas mais poderosas do regime.

Os estudos sobre nichos de inovação, inicialmente, enfocavam suas análises em atividades mercadológicas, especialmente aquelas ligadas a novas tecnologias. Recentemente, passou-se a discutir, também, formas de inovação que surgem de ações de base comunitária para o desenvolvimento sustentável. Unindo a literatura sobre nichos de inovação tecnológica com a literatura sobre ações comunitárias, Seyfang \& Smith (2007) criaram o conceito de grassroots innovation (inovações sociais de base), que seriam "redes de ativistas e organizações gerando novas soluções 'de baixo para cima' para o desenvolvimento sustentável; soluções que respondem à situação local e aos interesses e valores das comunidades envolvidas" (Seyfang \& Smith, 2007, p. 585, tradução nossa).

A PMN afirma que as ações sociais de base são loci de inovação que têm sido negligenciados pela literatura. Alguns nichos de inovação de base investigados são: sistemas energéticos comunitários (Hargreaves et al., 2013; Seyfang et al., 2013), casas sustentáveis (Seyfang, 2010), moedas sociais (Seyfang \& Longhurst, 2013), cidades em transição (Seyfang et al., 2010), tecnologias sociais (Smith et al., 2014) e ecovilas (Boyer, 2015). Outros exemplos citados de inovações sociais de base são as co-housings, fazendas cooperativas orgânicas, bancos de horas (Time Banks), entre outras.

Em contraste com as inovações sustentáveis de mercado desenvolvidas pelas empresas com o objetivo de gerar lucro, as inovações sociais de base operam nas arenas da sociedade civil e surgem em resposta a necessidades sociais e ambientais não atendidas. Dessa forma, essas inovações de base podem trazer benefícios onde medidas "de cima para baixo" não conseguem - graças ao seu conhecimento localizado sobre o que funciona e o que importa para os habitantes locais. Muitas das inovações geradas nessas iniciativas de base referem-se ao desenvolvimento de novas práticas sociais e novos significados culturais. Essas iniciativas questionam práticas cotidianas consideradas "normais" e as recriam coletivamente segundo uma lógica diferente, criando "nichos de novas normalidades" (Seyfang et al., 2010).

No entanto, os nichos de base enfrentam diversos desafios para poderem se estabelecer e manter suas atividades, tais como falta de recursos, de habilidades e de pessoal. Muitas dessas iniciativas se desfazem e o aprendizado desenvolvido por elas acaba se perdendo. Além disso, sua pequena escala, enraizamento cultural e choque de valores com as correntes dominantes tornam difíceis a sua replicação e a transferência de suas ideias e práticas (Seyfang \& Smith, 2007; Seyfang, 2010; Smith et al., 2014; Kemp et al., 2015). 
Segundo Seyfang (2010), a difusão de conhecimentos e práticas desenvolvidos nos nichos de inovação social de base podem se dar de três maneiras: replicação na mesma escala (crescimento de iniciativas similares, atração de novos participantes), aumento de escala ou "upscaling" (influência nas práticas de organizações de fora do nicho) e tradução de ideias para o regime dominante (quando surgem janelas de oportunidades no regime dominante, tal como iniciativas governamentais que encorajam a adoção de práticas de nichos). Vamos explorar esses caminhos de difusão mais adiante.

\subsection{Teoria das Práticas Sociais}

Recentemente, uma nova perspectiva tem sido apresentada para se estudar a inovação em direção à sustentabilidade: a TPS (Shove \& Walker, 2010; Spaargaren, 2011; Strengers, 2013; Shove et al., 2012; 2015; Boyer, 2016). Ao contrário das teorias clássicas sobre difusão de inovações, na TPS, a adoção e a manutenção de certas práticas são entendidas não como o resultado de uma decisão racional e intencional, mas como um processo fluido e rotinizado, ancorado em hábitos e formas de conhecimento prático que, em sua grande parte, não são conscientes (Bourdieu, 1995; Shove et al., 2012).

Nessa perspectiva, a análise enfoca os elementos simbólicos, corporais e materiais que condicionam a adoção de tecnologias e infraestruturas mais sustentáveis (Reckwitz, 2002; Shove \& Walker, 2010; Spaargaren, 2011). Dessa forma, a difusão de inovações passa a ser entendida dentro de um contexto cultural mais amplo, no qual os significados, representações, habilidades e conhecimentos tácitos se inter-relacionam com os sistemas de abastecimento, as infraestruturas e os objetos. Essa inter-relação gera tanto a reprodução das práticas sociais quanto a sua inovação. Não podem existir inovações técnicas sem uma correspondente inovação nas práticas. Os produtos e serviços, para ser adotados, devem estar integrados ao ordenamento da vida cotidiana.

Reckwitz (2002) define as práticas sociais como "um tipo rotinizado de comportamento que consiste de diversos elementos interconectados: formas de atividades corporais, formas de atividades mentais, 'coisas' e seus usos, um conhecimento contextual na forma de entendimento, know-how, estados emocionais e conhecimento motivacional" (p. 249, tradução nossa). Shove et al. (2012) sintetizam esses elementos em três categorias:

- materiais: que inclui coisas e tecnologias;

- competências: que inclui habilidades, know-how e conhecimento prático; e

- significados: que inclui significados simbólicos, ideias e aspirações.

Consideramos as formas de competências como um elemento corporal, por exigirem a incorporação de novos hábitos, condicionamentos e formas de conhecimento tácito (Lizardo, 2009; Bourdieu, 2012; Reckwitz, 2002; Roysen, 2015).

Segundo Shove et al. (2012), as "práticas emergem, persistem, mudam e desaparecem quando as conexões entre elementos desses três tipos são feitas, sustentadas ou quebradas" (p. 14, tradução nossa). As práticas, enquanto performances, são necessariamente localizadas: instantes situados de integração dos elementos. Elas só existem e persistem por causa das incontáveis atuações reproduzidas pelos indivíduos. As práticas, nesse sentido, não circulam. O que circulam são os elementos que as constituem e que permitem a sua reatuação em novos locais. Cada reatuação, por sua vez, transforma a prática e reconfigura as suas características. 
Shove \& Walker (2010) dão o exemplo da prática do banho diário. Essa prática não surge da somatória de inúmeras decisões racionais individuais. Ela emerge a partir de transformações nas trajetórias de seus elementos: mudanças nos conceitos de saúde, frescor e bem-estar, nos investimentos em fornecimento de água e novas tecnologias de chuveiro, na crescente oferta de xampus e produtos de higiene pessoal nos mercados etc. A associação entre esses elementos suscitou a emergência de novas convenções sociais e favoreceu a incorporação de novos hábitos pelos indivíduos em suas vidas cotidianas.

Da mesma forma, a adoção da prática da reciclagem envolve não somente uma decisão racional, mas competências (conhecimento sobre quais resíduos podem ser reciclados e a adoção de novos hábitos), materiais (uma infraestrutura de coleta seletiva, latas de lixo específicas para separação) e os significados associados à reciclagem. Na perspectiva das práticas sociais, a difusão da reciclagem, assim como a transformação de outras práticas sociais no sentido da sustentabilidade, depende tanto da agência (significados e competências incorporados pelos indivíduos) quanto da estrutura (por exemplo, a oferta de coleta pública seletiva), ou seja, a mudança das práticas combina dinâmicas de mudança "de baixo para cima" com as dinâmicas "de cima para baixo".

A perspectiva das práticas sociais enfatiza o papel dos praticantes na geração, sustentação e destituição das práticas diárias, sem cair num paradigma individualista. Acreditamos que a utilização do referencial da TPS em estudos sobre difusão em nichos de inovação social de base pode contribuir para o entendimento de como e por que certos grupos criam inovações radicais em suas práticas e como essas inovações podem ser difundidas para além desses nichos.

Os estudos sobre nichos, na PMN, tendem a usar como nível de análise o conjunto de organiza- ções existentes em um país (ou em vários países) e que participam de um determinado nicho, enfocando a história da sua evolução e sua difusão ao longo do tempo. No entanto, este ensaio sugere que, para podermos compreender melhor o processo de inovação e difusão de práticas sociais, devemos nos debruçar sobre estudos empíricos mais qualitativos, isto é, estudos que, a partir de etnografias e entrevistas em profundidade, atentem para o processo de formação dessas organizações que compõem os nichos, para as motivações individuais e coletivas que levam à inovação e para as inter-relações entre tecnologias, habilidades e significados na mudança das práticas sociais. Algumas perguntas norteadoras são: o que leva as pessoas a modificarem as suas práticas e se dedicarem à construção de um nicho? Qual é o papel das tecnologias, das habilidades e dos significados na transformação das práticas? Como se constitui um grupo com normas sociais divergentes das normas do regime?

Os nichos não podem ser entendidos como fenômenos dados, cuja origem não necessita de explicação. Ao contrário, é na formação das organizações dos nichos que encontraremos elementos para a compreensão mais profunda dos processos de inovação das práticas, assim como das possibilidades de intervenção social na direção da sustentabilidade.

\section{Estudando a mudança e a difusão das práticas em nichos de inovação social: o caso do movimento das ecovilas}

Vimos, na seção anterior, que a mudança das práticas cotidianas não somente envolve uma decisão racional com base nos atributos da nova prática (como propunham os estudo clássicos sobre difusão) como está intrinsecamente relacionada aos sistemas sociotécnicos dominantes (conforme 
propõe a $\mathrm{PMN}$ ): as pessoas organizam suas vidas em torno dos sistemas institucionalizados de transporte, saneamento e alimentação. Elas adaptam suas casas e seus estilos de vida a esses sistemas institucionalizados, tendendo a reproduzir as práticas que são consideradas "normais" e valorizadas pela sociedade em que vivem. Da mesma forma, as mudanças que surgem de "cima para baixo" também tendem a ser incrementais, melhorando os sistemas já instituídos, regularizados e normalizados de atender às necessidades sociais. Dessa forma, mudanças radicais nas práticas necessitam de um espaço protegido para ser desenvolvidas: são os chamados nichos de inovação social de base.

A TPS sugere que as práticas, enquanto performances, só existem quando são atuadas e reproduzidas pelas pessoas. Para que isso aconteça, os elementos materiais, corporais e simbólicos que as constituem devem estar disponíveis. Esses elementos têm trajetórias próprias e circulam de diferentes formas. Sugerimos que os nichos de base são importantes para a inovação das práticas por reunirem, em um mesmo lugar, diversos elementos que possibilitam a atuação de práticas sociais alternativas. Entre esses elementos estão: tecnologias, materiais e espaços propícios às novas práticas; significados, valores e normas alternativos; e novos hábitos e competências incorporados por seus membros. Vamos, agora, tentar explorar o potencial da TPS na análise da difusão em nichos de inovação social de base, usando como exemplo o caso do movimento das ecovilas.

\subsection{O movimento das ecovilas como nicho de inovação social de base}

A Rede Global de Ecovilas (Global Ecovillage Network) define uma ecovila como "uma comuni- dade intencional ou tradicional usando processos participativos para integrar de forma holística as dimensões ecológica, econômica, social e cultural da sustentabilidade, de forma a regenerar os meio ambientes sociais e naturais" (Gen, s.d., tradução nossa). Trata-se de grupos de pessoas que decidem viver e trabalhar juntas, experimentando novas formas de governança, habitação, consumo, educação, etc.

A vida em uma ecovila baseia-se em três dimensões interligadas: ecológica, social e cultural/ espiritual. A dimensão ecológica se manifesta em práticas locais sustentáveis, tais como bioconstrução, permacultura (Veteto \& Lockyer, 2008), tratamento ecológico dos resíduos domésticos (Swilling \& Annecke, 2006)solid waste removal, energy, building materials and food security.(1, valorização de sistemas energéticos alternativos, proteção da biodiversidade, preservação do solo e da água, entre outros (Borsos, 2013; Roysen, 2013; Kunze, 2015). O Instituto de Permacultura e Ecovilas do Cerrado (Ipec), por exemplo, desenvolveu um sanitário compostável que utiliza serragem no lugar de água na descarga sanitária. Conhecido como húmus sapiens ou banheiro seco, venceu em 2007 o Prêmio Finep de Inovação Tecnológica, na categoria Inovação Social (Capello, 2013).

A dimensão social corresponde ao desejo das pessoas de construírem relacionamentos de confiança e ajuda mútua. Nas ecovilas, seus membros tomam decisões e realizam trabalhos de forma coletiva. Essa dimensão se reflete em práticas de autogestão (tomada de decisão por consenso ou consentimento, liderança circular), de conexão emocional (técnicas de partilha, resolução de conflitos e feedbacks) e de integração social (almoços comunitários, atividades coletivas, rituais, compartilhamento de ferramentas e espaços etc.) (Kirby, 2003; Christian, 2007; Loezer, 2011; Roysen, 2013; Kunze, 2015b; Boyer, 2016). 
A dimensão cultural/espiritual varia muito de grupo para grupo. Existem ecovilas religiosas e seculares. No entanto, todas as ecovilas incentivam o autoconhecimento e a mudança interna como parte indissociável da mudança das práticas. Segundo os membros entrevistados na pesquisa de campo, o convívio intenso dentro de uma comunidade, bem como o compartilhamento de objetos e dos espaços, exige das pessoas um processo de desconstrução interna de conceitos e padrões de comportamento profundamente enraizados. Algumas questões recorrentes nas falas dos membros e que exigem essa desconstrução são: a ideia de posse, do "meu"; o conceito de privacidade; expectativas divergentes com relação à limpeza e à organização dos espaços coletivos; a necessidade de se abrir mão do seu jeito pessoal de fazer as coisas para aceitar o jeito dos outros; o equilíbrio entre ceder e se colocar durante os trabalhos coletivos; tomadas de decisões conjuntas com pessoas com visões de mundo e criações diferentes; entre outras. Essas são questões que, segundo eles, exigem das pessoas um trabalho de autoconhecimento e o desenvolvimento de valores considerados espirituais, tais como o desapego, a tolerância, o respeito e a autorresponsabilidade. Essa abertura para a desconstrução interna se reflete em uma abertura para experimentar novas práticas sociais.

As ecovilas têm sido descritas na literatura como "incubadoras de inovações sociais" e como "laboratórios de experimentações" (Jørgensen et al., 2015; Kunze, 2015a), pois são espaços em que são criados e desenvolvidos novos conhecimentos, práticas e tecnologias sociais em diversas áreas: na construção, no plantio, nas relações interpessoais, na estrutura organizacional, nas formas de tomada de decisão e resolução de conflitos, na organização social, no trabalho e no consumo (Capello, 2013;
Avelino, 2015; Boyer, 2015; Kunze, 2015b). Tudo isso a partir dos recursos dos próprios participantes, sem nenhuma forma de ajuda ou reconhecimento do governo. Trata-se, portanto, de um nicho de inovação de base: espaços protegidos em que alternativas radicais podem ser desenvolvidas. Nas ecovilas, essas práticas alternativas passam a ser consideradas "normais" e valorizadas pelo grupo. No entanto, assim como ocorre com diversos outros nichos de inovação social, as ecovilas enfrentam diversos desafios financeiros, legais e tecnológicos para sua sustentação (Kirby, 2003; Ergas, 2010) e mobilizam pouca legitimação social.

Nas ecovilas, assim como em outros nichos de base, as práticas surgem a partir de um conhecimento situado sobre as necessidades sociais e ambientais de cada lugar. Muitas inovações que esses grupos desenvolvem têm um caráter acentuadamente social (em vez de tecnológico). Uma das práticas estudadas na pesquisa de campo foi a dos carros compartilhados. Essa prática surgiu de uma necessidade real sentida pelos membros da ecovila: a distância da cidade e a falta de oferta de transporte público. As únicas possibilidades para seus membros se deslocarem da zona rural até a cidade são por bicicleta ou por carros. As bicicletas são adotadas pelos membros mais jovens e sem filhos. A maior parte das pessoas, no entanto, precisa de carros para se locomover. Dessa forma, o grupo conseguiu sistematizar uma prática de compartilhamento de carros de forma que todos pudessem ter acesso a esse meio de locomoção, sem que cada família precisasse ter o seu próprio carro, e que os custos fossem justamente partilhados entre usuários e proprietários. Esse é um ótimo exemplo de uma inovação de caráter social que surge, de baixo para cima, como uma resposta comunitária a um problema local percebido. 


\subsection{Inovação das práticas nas ecovilas}

As ecovilas, ao reunirem diversas práticas que visam à sustentabilidade, demonstram a possibilidade de um estilo de vida menos impactante ao meio ambiente. Na ecovila americana Dancing Rabbit, por exemplo, seus membros consomem menos de $10 \%$ da energia e dos recursos materiais de um norte-americano médio, em diversas categorias de consumo (Boyer, 2016). A criação e a integração dessas diversas práticas sustentáveis são possíveis graças a transformações nos elementos que compõem as práticas sociais: nos significados, nas competências e nos materiais. Vamos agora destrinchar o papel de cada elemento na inovação das práticas nas ecovilas.

Em primeiro lugar, nas ecovilas encontramos novos significados. Podemos incluir nessa categoria a crítica sobre os regimes sociotécnicos estabelecidos e, consequentemente, a motivação para buscarem práticas alternativas. Entre os significados presentes nesse nicho estão:

- reaproveitamento: mais importante do que ter objetos novos, os membros das ecovilas procuram reutilizar os materiais, o que os leva a novas convenções com relação a moradia, aparência, higiene e consumo (Roysen, 2013);

- responsabilidade pelos resíduos: existe uma preocupação com o destino dos resíduos e efluentes domésticos. Isso, necessariamente, leva a uma reavaliação do consumo (redução) e do ciclo de vida dos materiais e resíduos, que devem ser reinseridos na natureza (compostagem) ou na cadeia produtiva (reciclagem);

- simplicidade: buscam desvincular a simplicidade do conceito de "pobreza". Nas ecovilas, o reaproveitamento de materiais, assim como a simplicidade, a construção com barro, o manejo de resíduos - tudo isso é visto com orgulho, como uma forma de se estar vivendo melhor e contribuindo para a preservação dos recursos naturais (Roysen, 2013); e

- comunidade: buscam incentivar atividades coletivas, tanto como forma de integração social como de redução da pegada ambiental. Isso se expressa em refeições preparadas coletivamente, compras coletivas, compartilhamento de ferramentas, carros e espaços, bem como em inúmeras reuniões para tomadas de decisão. Seus membros reconhecem que a horizontalidade na tomada de decisões e no trabalho faz que as ações levem muito mais tempo e gerem muito mais conflitos do que em uma estrutura hierárquica. No entanto, acreditam que a "inteligência coletiva" gera soluções mais criativas.

As práticas desenvolvidas pelas ecovilas também são constituídas por elementos materiais, o que inclui novas tecnologias e infraestruturas, tais como composteiras, banheiros secos, tecnologias de energias renováveis, casas coletivas, cozinhas comunitárias etc. Essa é uma dimensão importante, pois os objetos e espaços possuem um papel constitutivo na vida cotidiana. Ao oferecer essa infraestrutura e uma organização do espaço adequados às suas práticas, a ecovila facilita a sua adoção/reprodução pelos membros. Os espaços são, portanto, "mais do que contextos ou cenários nos quais as performances se dão. Em certas situações, a colocalização resulta em novas formas híbridas: práticas novas emergem" (Shove et al., 2012, p. 124, tradução nossa). 
Por fim, os membros das ecovilas precisam desenvolver, também, novas competências. Entre elas, o conhecimento tácito sobre o funcionamento dessas infraestruturas, o aprendizado sobre plantio e manejo da terra (visto que a maior parte dessas pessoas vem de ambientes urbanos) e competências sociais (especialmente a adaptação ao convívio intenso que ocorre na comunidade). Tudo isso exige a incorporação dos esquemas motores necessários para o desenvolvimento desses novos hábitos e habilidades práticas (Lizardo, 2009; Roysen, 2015).

Estudos futuros podem investigar qual é o peso de cada um desses elementos na adoção de novas práticas. Quais elementos são mais fáceis de ser incorporados? Como a circulação desses elementos pode ser incentivada por meio de políticas públicas? Sugerimos que, no caso do movimento das ecovilas, o primeiro elemento a ser incorporado é o elemento simbólico - uma afinidade ideológica com os símbolos e significados promovidos pelo movimento. Em sua maior parte, os indivíduos decidem viver em uma ecovila por apresentarem um questionamento intelectual e uma sensação de desconforto para com os regimes sociotécnicos dominantes e as formas de relação interpessoal que esses regimes produzem. Esse questionamento leva as pessoas a buscarem alternativas e entrarem em contato com o movimento das ecovilas.

Algumas pessoas entrevistadas na pesquisa de campo relatam tentativas frustradas de adotar práticas mais sustentáveis quando viviam na cidade - práticas que não conseguiram manter por encontraram barreiras materiais (falta de espaço e/ou materiais adequados) ou normativas (falta de apoio moral das pessoas ao redor). Essas tentativas frustradas de adoção de práticas inovadoras fora de um nicho se relacionam com o que a PMN chama de "mecanismos de trancamento" associados aos regimes sociotécnicos existentes na nossa sociedade.
Por exemplo, a instalação de um banheiro seco ou de um tratamento de efluentes alternativo (como, por exemplo, um “círculo de bananeiras") esbarra em mecanismos de trancamento criados pelo regime de saneamento dominante: dificuldades legais (falta de uma legislação específica, dificuldade de aprovação pela agência de vigilância sanitária), dificuldades simbólicas (associação do banheiro seco com sujeira e contaminação, normas sociais desfavoráveis), dificuldades materiais (dificuldade de encontrar materiais apropriados e espaços construídos adaptados) e problemas de competência (falta de conhecimento técnico e tácito para a sua implantação, necessidade de adaptação dos estilos de vida).

Ao viver em uma ecovila, o indivíduo consegue superar essas barreiras e encontrar um terreno fértil para a experimentação com novas práticas. Apesar de haver muitos grupos de permacultura urbana, grande parte da experimentação com bioconstrução e sistemas alternativos de água e tratamento de efluentes ocorre na zona rural, justamente pela carência de regulamentação e fiscalização nessas áreas mais distantes.

Dessa forma, podemos sugerir que, após se alinhar com os significados do movimento, o segundo elemento no processo de adoção é o material. Somente com o tempo é que as competências vão sendo incorporadas e as novas práticas tornam-se hábitos, facilitadas pela presença de pessoas mais experientes no nicho. Os membros da ecovila estudada afirmam que as competências sociais e as relacionadas ao compartilhamento são muito mais difíceis de ser adquiridas do que as competências relacionadas a técnicas específicas de plantio, compostagem ou bioconstrução.

O desenvolvimento de novas práticas nos nichos é um processo coletivo que envolve inúmeras tentativas e erros. Elas exigem tempo para ser 
aperfeiçoadas e incorporadas por todos os membros. Além da conexão entre os elementos para a criação de novas práticas, as práticas também se conectam entre si para formar padrões regulares. Shove et al. (2012) chamam esses agrupamentos de "pacotes" (padrões mais soltos baseados na colocalização e na coexistência) ou "complexos" (arranjos mais integrados, incluindo formas de sequência e sincronização).

Vamos dar o exemplo da prática do "cuidado coletivo", realizada na ecovila estudada na pesquisa de campo. Trata-se de um esquema de rodízio das funções de limpeza e preparo dos alimentos. Em cada dia da semana existe uma equipe de três ou quatro membros responsáveis por preparar o café da manhã, o almoço e o jantar para os 30 moradores, varrer o espaço coletivo, levar os resíduos recicláveis para um galpão, levar os resíduos orgânicos para as composteiras, preparar as bacias da lavagem permacultural (destinadas à lavagem dos pratos e talheres com economia de água), entre outras tarefas. Essa prática se constitui, portanto, como um complexo de outras práticas menores que a compõem. O dia de "cuidado coletivo" é um dia intenso de trabalho. Em compensação, nos outros dias, os membros são servidos pelas outras equipes.

A prática do cuidado coletivo é ambientalmente sustentável, pois diminui a quantidade de resíduos gerados. Quando as refeições são preparadas e consumidas coletivamente, necessariamente, surge a necessidade de fazer as compras de forma também coletiva e de discutir, em conjunto, quais alimentos entram ou não na lista de compras. Dessa forma, os produtos são comprados em grandes quantidades, requerendo menos embalagens e menos viagens ao mercado do que exigiriam compras individuais. Além disso, existe uma ampla discussão interna sobre a sustentabilidade dos alimentos consumidos, e grande parte deles é comprada diretamente de assentamentos que trabalham com produção agroecológica. A prática do cuidado coletivo também é economicamente sustentável, pois se gasta menos com compras e uso de gás e água. Com 180 reais por mês, os membros têm o direito de consumir três refeições diárias preparadas, em grande parte, com alimentos orgânicos. Por fim, essa prática também é socialmente sustentável, gerando integração social entre os membros da comunidade. Os visitantes também entram no rodízio, facilitando a sua integração ao grupo. Ao participarem dessa prática, eles entram em contato com as diversas práticas sustentáveis que compõem o cuidado coletivo: o manejo dos resíduos orgânicos, a triagem dos resíduos recicláveis, a lavagem permacultural, a colheita de alimentos na horta, entre outras.

Os membros relatam que, no processo de desenvolvimento dessa prática, diversos modelos foram tentados até se chegar à sua forma atual. $\mathrm{O}$ esquema é constantemente reavaliado pelos membros e modificado para atender à situação atual dos participantes. Nas reuniões, os membros reavaliam as atividades que entram no cuidado coletivo, os alimentos que entram ou não na lista de compras coletivas, as relações com assentamentos e outros fornecedores, o formato do rodízio, a configuração das equipes, entre outros elementos. Diversas ideias foram trazidas pelos participantes com base em suas experiências prévias em outros espaços coletivos - em especial, a experiência em repúblicas universitárias e na vivência familiar. Significados associados a questões de gênero e trabalho tiveram que ser reformulados pelos membros. Novas competências tiveram que ser incorporadas, tais como cozinhar para um grande número de pessoas e realizar trabalhos cotidianos em equipe. Dessa forma, as práticas na ecovila nunca são completamente estáveis. Elas estão sempre sendo questionadas, reformuladas e reconfiguradas de diferentes for- 
mas. Trata-se de arranjos desenvolvidos ao longo do tempo, com base nas necessidades do grupo e a partir de inúmeras tentativas e erros, e que vão incorporando elementos e técnicas trazidos pelas pessoas de outras vivências.

Outro exemplo de integração e interdependência entre práticas diversas na ecovila é o complexo que se forma em torno da compostagem. A utilização do banheiro seco, ao mesmo tempo em que permite uma economia de água por não utilizar descarga, gera um resíduo compostável. Da mesma forma, a separação dos resíduos orgânicos na cozinha também leva à necessidade de práticas de compostagem para lidar com esses materiais. A produção do composto está ligada às práticas de plantio e manejo da terra, por fornecer adubação para a produção orgânica, que, por sua vez, abastece a cozinha. Essas práticas formam, assim, um complexo, no qual uma prática é indispensável para a outra.

Segundo Shove et al. (2012), elementos compartilhados, tais como colocalização, significados e sistemas de infraestrutura, constituem padrões importantes de associação entre as práticas. Podemos dizer que os nichos de inovação de base criam pacotes e complexos de práticas que estão interligadas e que, muitas vezes, compartilham os mesmos significados, materiais ou competências. Além disso, as formas de competência geradas no desenvolvimento de uma prática podem migrar para o desenvolvimento de novas práticas. A prática do cuidado coletivo permitiu aos membros da ecovila a incorporação de novas competências relacionadas ao trabalho em grupo e à tomada de decisão coletiva, bem como o desenvolvimento da confiança entre os membros. Essas competências sedimentadas pelo cuidado coletivo abriram caminho para o desenvolvimento de novas práticas coletivas, como os carros compartilhados. O bom funcionamento da prática dos carros compartilhados, por sua vez, tem encorajado os membros a pensarem no subsequente desenvolvimento dessa prática (por meio da compra de um carro propriamente coletivo), bem como no desenvolvimento de novas práticas que se orientam para uma maior coletivização, tais como uma lavanderia coletiva e um caixa coletivo.

Cada prática coletiva se constrói sobre competências previamente adquiridas e significados compartilhados pelo grupo, levando a um desenvolvimento incremental de práticas sociais alternativas. Os nichos favorecem, assim, a criação de novos "circuitos de reprodução" (Giddens, 2007). O contínuo monitoramento que os agentes têm sobre a sua ação fornece feedback sobre os resultados das atuações anteriores, alimentando ações futuras. $\mathrm{Na}$ ecovila estudada, as reuniões semanais criam um compartilhamento desses feedbacks, tornando o monitoramento e a mudança das práticas um processo não só individual como, também, coletivo. Assim como os regimes sociotécnicos dominantes tendem a orientar os possíveis caminhos de desenvolvimento das práticas, dificultando mudanças radicais, os complexos de práticas criados nos nichos delimitam um novo campo de possibilidades para o desenvolvimento das práticas, de acordo com os elementos ali disponíveis.

\subsection{Adoção das práticas nas ecovilas}

Diversos estudos sobre a TPS e sobre difusão de inovações têm ressaltado a importância das normas sociais na adoção de novas práticas (Rogers, 1995; Lindt \& Ozaki, 2011; Spaargaren, 2011; Seyfang \& Haxeltine, 2012; Shove et al., 2012; Smith \& Raven, 2012; Emmert \& Luiten, 2013). Devido à sua importância, buscamos explorar um pouco mais esse conceito. 
A incorporação de uma nova prática social é facilitada quando existe uma norma social favorável. Quando uma prática é vista como "normal" e é valorizada pelo grupo do qual fazemos parte, sentimos uma pressão social para realizá-la. As normas sociais são regras e padrões de comportamento compartilhados pelos membros de um grupo, que podem ou não ser explicitamente declarados. Esses padrões guiam e/ou limitam o comportamento social sem a força de leis (Cialdini \& Trost, 1998). O comportamento dos outros nos fornece informação consensual: "Quanto maior o número de pessoas que respondem à mesma situação da mesma maneira, mais perceberemos tal comportamento como correto" (Cialdini \& Trost, 1998, p. 155, tradução nossa).

Quando um determinado comportamento que tem impacto ambiental é percebido como uma norma social pelo indivíduo, este tende a agir em conformidade com a norma percebida (Goldstein et al., 2008; Nolan et al., 2008; Schultz et al., 2007). Um estudo realizado no Reino Unido, por exemplo, identificou um aumento no número de indivíduos que alegam reciclar regularmente, de forma que a reciclagem se tornou um comportamento normal que a maior parte das pessoas faz. Dessa forma, a "pressão social" exercida pelo conhecimento de que os outros estão reciclando tem tido um efeito positivo no comportamento geral com relação à reciclagem, possibilitando o estabelecimento de novas normas sociais (Thomas \& Sharp, 2013, p. 17, tradução nossa).

É preciso um alto grau de independência para que um indivíduo consiga manter práticas e atitudes que contrariem as normas predominantes em seu meio social (Asch, 1977). Daí a importância dos nichos para o desenvolvimento de novas práticas: eles criam espaços de "novas normalidades", nos quais as pessoas compartilham uma forma inovadora de pensar e agir e, portanto, obtêm no grupo apoio moral para adotar e manter essas novas práticas.

Dessa forma, viver em uma ecovila favorece a adoção e a incorporação de práticas sustentáveis, pois o grupo exerce pressão nos indivíduos para a adoção dessas práticas e para o cumprimento de certos padrões de comportamento (Roysen, 2013). A ecovila cria um nicho de "novas normalidades" e novas regras de conduta. A criação de soluções inovadoras para os problemas locais é valorizada nesses nichos e existe abertura para o erro - características que estimulam a experimentação.

Essa abertura para a experimentação inclui as contribuições feitas pelos visitantes. Um exemplo retirado da ecovila estudada é o "piscilago". Segundo os membros, eles já tinham a ideia de transformar a piscina da casa coletiva (que já existia quando a terra foi comprada) em um lago. Mas foi um visitante que viveu com eles durante um ano que os ajudou a implementar essa ideia. Colocaram peixes e plantas aquáticas na piscina, transformando-a em um sistema que se autorregula e se mantém próprio para banho sem a necessidade de produtos químicos ou cuidados constantes. Seus membros afirmam que essa receptividade a novas ideias - mesmo de pessoas que estão apenas transitando pela ecovila - os torna um polo receptor e emissor. Muitos visitantes trazem novas ideias para o grupo, muitas vezes aprendidas em outras comunidades. Essa circulação de viajantes entre as ecovilas tem se mostrado, na nossa pesquisa, como um fator importante de difusão de inovações entre as organizações desse mesmo nicho.

Um ponto importante a ser ressaltado é que, ao se envolverem com uma ecovila e assumirem compromissos para com a sua comunidade, as vidas das pessoas passam a girar em torno desse nicho: a ecovila se torna um "projeto dominante" (Shove et al., 2012). Os projetos dominantes enfocam o 
tempo e a energia das pessoas em algumas direções e não em outras, o que faz que os membros dos nichos desenvolvam habilidades e competências que, muitas vezes, só são reconhecidas no interior desse mesmo nicho. A vida em uma ecovila exige, portanto, um alto nível de comprometimento, sendo uma mudança radical na vida das pessoas. Esse choque entre as práticas e valores do nicho e do regime se expressa na relação dos membros com os seus familiares que não participam do nicho e que, na maior parte das vezes, apresentam dificuldades em compreender e aceitar esse novo estilo de vida.

\subsection{Difusão das práticas nas ecovilas}

Segundo Seyfang (2010), a difusão de conhecimentos e práticas desenvolvidos nos nichos de inovação social de base pode se dar de três maneiras: replicação na mesma escala, aumento de escala e tradução de ideias para o regime dominante. A replicação corresponde à adoção de práticas desenvolvidas nas ecovilas pelos indivíduos em seu cotidiano, podendo resultar ou não na sua decisão por viver em uma ecovila. Muitas ecovilas criam centros educacionais, oferecendo cursos e vivências, e recebem centenas de visitantes e voluntários todos os anos. Por meio desses cursos e vivências, novas pessoas passam a entrar em contato com os elementos presentes nas ecovilas, podendo adotar algumas de suas práticas. Cabe ressaltar que as práticas aprendidas nas ecovilas, ao serem replicadas em outros lugares, serão, necessariamente, reinventadas de acordo com cada contexto e cada grupo.

Muitos visitantes chegam à ecovila já alinhados com a sua ideologia, em busca de aprendizados técnicos sobre manejo da terra, agroflorestas, bioconstrução e vida comunitária. Nesses casos, a ecovila oferece uma vivência prática na qual novas habilidades e conhecimentos podem ser adquiridos. Outros visitantes chegam à ecovila e entram em contato com a ideologia do movimento pela primeira vez. Nesses casos, a difusão dos elementos simbólicos se mostra importante por ser, potencialmente, o primeiro passo para a futura adoção de práticas sustentáveis pelos indivíduos. Sugerimos que o conceito de identidade coletiva desenvolvido pelos estudiosos dos movimentos sociais pode ser interessante para se pensar em como esses significados se difundem entre (e para fora de) organizações de um mesmo nicho.

Polletta \& Jasper (2001) conceituam identidade coletiva como "a conexão cognitiva, moral e emocional de um indivíduo com uma comunidade, categoria, prática ou instituição. É a percepção de um status ou relação compartilhada, que pode ser imaginada mais do que diretamente vivenciada" ( $p$. 285, tradução nossa). Quanto mais importante uma identidade coletiva é para a identidade pessoal do indivíduo, mais ele tenderá a seguir as normas sociais associadas a essa identidade como guia para a ação. Dessa forma, ao se identificar com as práticas e ideologias de determinado nicho, o indivíduo se sente parte de um movimento mais amplo e tem um incentivo moral para transformar seus hábitos e adotar práticas mais sustentáveis.

O movimento das ecovilas tem criado uma identidade coletiva que vai além das ecovilas isoladas. A comunicação globalizada, a criação de redes transnacionais e a presença de indivíduos que viajam pelas ecovilas difundindo ideias e inovações permitem ao movimento criar um senso de identidade compartilhada (Salazar, 2013). A consolidação de uma identidade coletiva pode transformar as ecovilas em um grupo de referência normativa com o potencial de guiar o comportamento de pessoas, mesmo das que não participam diretamente do movimento. 
As ecovilas oferecem ao público mais amplo a oportunidade de cursos, vivências e programas de voluntariado in loco. Pesquisas futuras poderiam explorar qual é o impacto dessas vivências nas identidades e práticas sociais dos participantes. As práticas aprendidas são adotadas e mantidas no cotidiano dos participantes? As ecovilas tornam-se um grupo de referência normativa para os indivíduos? Qual é a influência da composição da rede social desses participantes na adoção e manutenção dessas práticas? Podemos sugerir que indivíduos imersos em redes pessoais com valores e normas sociais condizentes tendem a adotar as práticas das ecovilas mais facilmente do que os indivíduos cujas redes pessoais apresentam valores e normas conflitantes. Por outro lado, a vivência na ecovila pode levar os indivíduos a reconfigurarem suas redes pessoais, passando a buscar novas relações com pessoas que compartilham dos mesmos valores.

A segunda forma de difusão das práticas dos nichos de base é o aumento de escala (upscaling), que ocorre quando as práticas de um nicho passam a influenciar organizações de fora do nicho. Isso pode ocorrer por meio de parcerias entre ecovilas e organizações mainstream. Um exemplo dado por Boyer (2015) é a parceria realizada entre a Ecovila de Ithaca, nos Estados Unidos, e o Ithaca College em um projeto chamado Learn@EVI. Esse programa, que incluiu o desenvolvimento conjunto de um currículo em "ciência da sustentabilidade", motivou o surgimento de diversas iniciativas afins, tais como o Ithaca Car Share (uma organização sem fins lucrativos que oferece acesso ao uso de carros compartilhados) e o Groundswell Center for Local Food and Farming, que oferece formação, acesso a recursos e incubação aos agricultores para a criação de sistemas de alimentação locais e sustentáveis. Acreditamos que existe um grande potencial, ainda inexplorado no Brasil, de formação de parcerias en- tre ecovilas e universidades. As ecovilas oferecem um ambiente receptivo a experimentos com tecnologias alternativas em fase de desenvolvimento.

Por fim, a terceira via de difusão de práticas é a tradução para o regime dominante. Isso ocorre quando suas práticas são adaptadas e adotadas pelos regimes sociotécnicos em níveis institucionais mais altos. Um exemplo de tradução é um prêmio dado pela Agência de Proteção Ambiental dos Estados Unidos, em 2011, a um projeto feito em parceria entre a Ecovila de Ithaca e o município de Tompkins para traduzir os conceitos da ecovila em práticas de zoneamento e no desenvolvimento de novas habitações (Boyer, 2015). Esse tipo de tradução envolve, portanto, a combinação de iniciativas "de cima para baixo" com as iniciativas de base já existentes.

Existem diversas barreiras na tradução das práticas dos nichos para os regimes. Uma delas é o fato de que as práticas desenvolvidas nos nichos tendem a ser apropriadas aos contextos locais. Dessa forma, para serem reproduzidas em novos lugares, elas requerem uma adaptação ao novo contexto. Essa falta de aplicabilidade globalizada dificulta a adoção dessas práticas de forma massiva. Smith et al. (2014) sugerem que, em vez de se abordar os nichos de base como provedores de moldes para a ação, os analistas devem refletir sobre quais atividades - tais como encubação, financiamentos e parcerias - poderiam favorecer a emergência de contextos propícios para a inovação de base.

Outra barreira na tradução é o conflito de valores geralmente presente entre nichos e regimes. Devido a esse conflito, a tradução tende a ser sempre parcial, resultando na perda de muitos dos valores mais profundos estimados pelos nichos (Seyfang, 2010). Isso porque as práticas dos nichos são interpretadas, adaptadas e acomodadas no regime em uma relação desigual de poder, na qual os regimes possuem uma longa história de interação entre 
tecnologias, usuários, conhecimento e instituições (Smith, 2007). Smith (2007) dá o exemplo do nicho de alimentos orgânicos, cujo rápido crescimento, na década de 1990, atraiu o interesse da indústria convencional de alimentos. O regime dominante absorveu apenas os elementos do nicho que eram adaptáveis às práticas vigentes, distanciando-se dos ideais mais radicais. Um exemplo disso são os equivalentes orgânicos de comidas altamente processadas - tais como comidas prontas congeladas - que surgiram no mercado. Ao mesmo tempo em que essa tradução para o regime atenuou a sustentabilidade orgânica, ela levou os produtos orgânicos para muito mais pessoas do que o nicho original poderia.

Segundo Boyer (2015), as organizações mais intermediárias dentro de um nicho são as que oferecem mais chances de traduzir as suas práticas para o regime dominante, ou seja, iniciativas que conseguem manter um equilíbrio entre os valores e práticas radicais do nicho, de um lado, e os valores e práticas convencionais do regime, do outro, estão em melhor posição para firmar parcerias com instituições e autoridades públicas e, assim, traduzir suas inovações para o regime. Essa propriedade de equilíbrio é chamada por Boyer de intermediaridade. Dessa forma, podemos esperar que ecovilas mais radicais e autocentradas tenham menos chances de influenciar mudanças nos regimes do que as ecovilas que estão mais disponíveis para a colaboração com atores externos, mesmo que isso signifique uma adaptação nas suas práticas e um conflito com seus valores mais profundos.

\section{Conclusão}

A Teoria da Prática Social (TPS) destaca a relação entre as práticas sociais e os objetos, infraestruturas, significados e competências existentes em um grupo. Ela traz uma nova abordagem ao estudo da difusão de inovações ao enfatizar o papel dos elementos simbólicos, cognitivos e materiais na reprodução e/ou transformação das práticas. Ao unir a TPS com a perspectiva multinível (PMN), entendemos que a criação de práticas sociais sustentáveis e inovadoras tende a ocorrer em nichos protegidos, nos quais novas "normalidades" são instituídas, novas competências podem ser desenvolvidas e nos quais são criados novos espaços e tecnologias. O estudo da inovação das práticas nesses nichos pode ajudar a iluminar o papel de cada elemento na difusão de práticas mais sustentáveis e quais intervenções podem incentivar a circulação desses elementos.

O estudo da inovação e difusão de práticas sociais sustentáveis, na perspectiva da TPS, é um campo ainda pouco explorado, mas extremamente valioso na busca por novas soluções para o desenvolvimento sustentável. Soluções tecnicistas e baseadas no mercado esbarram em diversos mecanismos de trancamento. Soluções mais radicais para a sustentabilidade são desenvolvidas em nichos de inovação social de base, apesar da falta de investimentos e políticas públicas. Torna-se importante, também, investigar as maneiras pelas quais o Estado pode apoiar e incentivar o desenvolvimento desses nichos não lucrativos e não mercadológicos de inovação. 


\section{Referências}

Asch, S. Psicologia Social. São Paulo: Editora Nacional, 1977.

Avelino, F. Transformative Social Innovation Narrative of Tamera Ecovillage. A Summary. TRANSIT: EU SSH.2013.3.2-1 Grant agreement $n^{\circ} 613169$ (613169), 2015.

Borsos, B. The Eco-Village Concept in a Model Experiment in South-West Hungary. Journal of Settlements and Spatial Planning 4(1), 2013.

Boulay, M.; Thomas W. V. The Relationship of Social Affiliation and Interpersonal Discussion to Family Planning Knowledge, Attitudes and Practice. International Family Planning Perspectives, 25(3), 112-138, 1999.

Bourdieu, P. Outline of a Theory of Practice. Cambridge: Cambridge University Press (livro eletrônico), 1995.

Bourdieu, P. O poder simbólico. Rio de Janeiro: Bertrand Brasil, 2012.

Boyer, R. Achieving One-Planet Living through Transitions in Social Practice: A Case Study of Dancing Rabbit Ecovillage. Sustainability: Science, Practice \& Policy, 12(1), 1-6, 2016.

Boyer, R. H. W. Grassroots Innovation for Urban Sustainability: Comparing the Diffusion Pathways of Three Ecovillage Projects. Environment and Planning A, 47(2), 320-337, 2015.

Cajaiba-Santana, G. Social Innovation: Moving the Field Forward. A Conceptual Framework. Technological Forecasting \& Social Change, 82, 42-51, 2014. doi: 10.1016/j. techfore.2013.05.008.

Capello, G. Meio ambiente e ecovilas. São Paulo: Senac, 2013.

Ceschin, F. Critical Factors for Implementing and Diffusing Sustainable Product-Service Systems: Insights from Innovation Studies and Companies' Experiences. Journal of Cleaner Production, 45, 74-88, 2013.

Choi, H.; Kim, S-H.; Lee, J. Role of Network Structure and Network Effects in Diffusion of Innovations. Industrial Marketing Management, 39(1), 170-177, 2010.
Christian, D. L. Starting a New Ecovillage: 'structural Conflict' \& Nine Ways to Resolve It. In:Joubert and Alfred (Eds.). Beyond you and me: inspirations and wisdom for building community. Hampshire: Permanent Publications, 2007. p. 49-57.

Cialdini, Rb.; Trost, Mr. Social Influence: Social Norms, Conformity and Compliance. The Handbook of Social Psychology, 2, 151-192, 1998. Disponível em: <http://psycnet. apa.org/psycinfo/1998-07091-021>.

Coleman, J. S.; Katz, E.; Menzel, H. The Diffusion of an Innovation Among Physicians. Sociometry, 20(4), 253270, 1957.

Ergas, C. A Model of Sustainable Living: Collective Identity in an Urban Ecovillage. Organization \& Environment, 23(1), 32-54, 2010.

Ester, P.; Vinken, H.; Simões, S. Cultural Change and Environmentalism: A Cross-Cultural National Approach of Mass Publics and Decision Makers. Ambiente \& Sociedade, VII(2), 45-66, 2004.

Furlanetto, E. L.; Santos, E. D. Difusão de inovações sustentáveis : o caso do biodiesel de mamona no Estado da Paraíba. Teoria e Prática em Administração, 4(1), 78103, 2014.

Geels, F. W. Ontologies, Socio-Technical Transitions (to Sustainability), and the Multi-Level Perspective. Research Policy, 39(4), 495-510, 2010. doi: 10.1016/j. respol.2010.01.022

Geels, F. W.; Schot, J. Typology of Sociotechnical Transition Pathways. Research Policy, 36(3), 399-417, 2007.

GEN - Global Ecovillage Network. n.d. What Is an Ecovillage? Disponível em: <http://gen.ecovillage.org/en/article/ what-ecovillage> . Acesso em: nov. 2015.

Giddens, A. The Constitution of Society: Outline of the Theory of Structuration. Cambridge: Polity Press, 2007.

Goldstein, N. J.; Cialdini, R. B.; Griskevicius, V. A Room with a Viewpoint: Using Social Norms to Motivate Environmental Conservation in Hotels. Journal of Consumer Research, 35(3), 472-482, 2008. doi: 10.1086/586910 
Granovetter, M. The Strenght of Weak Ties. American Journal of Sociology, 78(6), 1360-1380, 1973.

Hargreaves, T.; Hielscher,S.; Seyfang, G.; Smith, A. Grassroots Innovations in Community Energy: The Role of Intermediaries in Niche Development. Global Environmental Change, 23(5), 868-880, 2013. doi: 10.1016/j. gloenvcha.2013.02.008

Hoppe, T. Adoption of Innovative Energy Systems in Social Housing: Lessons from Eight Large-Scale Renovation Projects in The Netherlands. Energy Policy, 51, 791-801, 2012. doi: 10.1016/j.enpol.2012.09.026

Jørgensen, M. S.; Dorland, J.; Pel, B.; Wittmayer. J. Transformative Social Innovation Theory Project: Characterisation and Comparison of Case Study Findings - Batch 1 Cases, 2015. Disponível em: < http://www.transitsocialinnovation.eu/content/original/Book $\% 20$ covers/Local $\% 20$ PDFs/157\%20D4\%202\%20Comparative\%20analysis \%20 17\%2004\%202015.pdf>.

Kaiser, F.; Wolfing, S.; Fuhrerm, U. Environmental Attitude and Ecological Behaviour. Journal of Environmental Psychology, 19, 1-19, 1999.

Kemp, R. et al. Doing Things Differently: Exploring Transformative Social Innovation and Its Practical Challanges, 2015. Disponível em: <http://www.transitsocialinnovation. eu/content/original/TRANSIT brief final_no bleed.pdf $>$.

Kemp, R.; Schot, J.; Hoogma, R. Regime Shifts to Sustainability through Processes of Niche Formation: The Approach of Strategic Niche Management. Technology Analysis \& Strategic Management, 10(2), 175-198, 1998. doi: $10.1080 / 09537329808524310$

Kimura, H.; Kazuo Kayo, E.; Jacob Perera, L. C. Difusão de inovações entre consumidores conectados em redes sociais. Revista Brasileira de Inovação, 10(1), 73-100, 2011.

Kirby, A. Redefining Social and Environmental Relations at the Ecovillage at Ithaca: A Case Study. Journal of Environmental Psychology, 23(3), 323-332, 2003.

Krasny, M. E. et al. Civic Ecology Practices: Insights from Practice Theory. Ecology \& Society, 20(2), 12, 2015.

Kunze, I. Ecovillages: Isolated Islands or Multipliers of Social Innovations? TRANSIT website, 2015a. Disponível em: $<$ http://www.transitsocialinnovation.eu/blog/ecovillages-isolated-islands-or-multipliers-of-social-innovations?utm source $=$ subscribers\&utm_campaign $=640 \mathrm{~b} 37 \mathrm{~d} 940$-TRANSIT_Newsletter_November_201511_2_2015\&utm me dium $=$ emai $1 \&$ t m_term $=0$ _d 7 f7 bd $8502-$ -640b37d940-2644933>.Acesso em: nov. 2015.

Kunze, I. Transformative Social Innovation Narrative of the Ecovillage of Schloss Tempelhof (TH). TRANSIT: EU SSH.2013.3.2-1 Grant agreement $n^{\circ}$ 613169, 2015 b. Disponível em: <http://www.transitsocialinnovation.eu/ content/original/Book $\% 20$ covers/Local $\% 20 \mathrm{PDF} / 147 \% 20$ TSI\%20Narrative_GEN_TH_Upload.pdf $>$.

Läpple, D.; Van Rensburg, T. Adoption of Organic Farming: Are There Differences between Early and Late Adoption? Ecological Economics, 70(7), 1406-1414, 2011.

Lindt, M van der; Emmert, S.; Luiten, H. Identifying Relevance and Strengh of Barriers to Change in Energy Behaviour among End Consumers and Households: The BarEnergy Project. In: Fudge, S.; Peters, M.; Hoffman, S. M.; Wehrmeyer, W.(Orgs.). The Global Challenge of Encouraging Sustainable Living: opportunities, barriers, policy and practice. Glos: Edward Elgar Publishing Limited, 2013. p. 106-130.

Lizardo, O. Is a ‘Special Psychology’ of Practice Possible? From Values and Attitudes to Embodied Dispositions. Theory \& Psychology, 19(6), 713-727, 2009.

Lockyer, J. From Earthships to Strawbales Sustainable Housing in Ecovillages. Anthropology News (December), 2008 .

Loezer, L. Enhancing Sustainability at the Community Level: Lessons from American EcoVillages. Master in Science in Architecture, University of Cincinnati, 2011. Disponível em: <https://etd.ohiolink.edu/ap:10:0:::10:P10_ACCESSION_NUM:ucin1321368949>. Acesso em: nov. 2014.

Mani, S.; Dhingra, T. Diffusion of Innovation Model of Consumer Behaviour - Ideas to Accelerate Adoption of Renewable Energy Sources by Consumer Communities in India. Renewable Energy, 39(1), 162-165, 2012.

Mertens, F.; Saint-Charles, J.; Mergler, D. Social Communication Network Analysis of the Role of Participatory Research in the Adoption of New Fish Consumption Behaviors. Social Science \& Medicine, 75(4), 643-650, 2012.

Moreira, M. F.; Vargas, E. R. de. Indução de inovações em serviços: compras governamentais para a inovação ou 
compras de inovações?" Revista Gestão \& Tecnologia, 15(2), 186-210, 2015.

Moura, L. R. C.; Cunha, N. R. S.; Porto, G. D.; Moura, L. E. L. de; Pires, R. R. Desenvolvimento de uma nova versão de escala para mensuração das características que incentivam a adoção de novos produtos: um estudo sobre a água mineral. Navus, 5(4), 96-112, 2015.

Nolan, J. M.; Schultz, P. W.; Cialdini, R. B.; Goldstein, N. J.; Griskevicius, V. Normative Social Influence Is Underdetected. Personality and Social Psychology Bulletin, 34(7), 913-923, 2008. doi: 10.1177/0146167208316691

Ozaki, R. Adopting Sustainable Innovation: What Makes Consumers Sign up to Green Electricity? Business Strategy and the Environment, 20(1), 1-17, 2011.

Polletta, F.; Jasper, J. M. Collective Identity and Social Movements. Annual Review of Sociology, 27, 283-305, 2001.

Ramani, S. V.; SadreGhazi, S.; Duysters, G. On the Diffusion of Toilets as Bottom of the Pyramid Innovation: Lessons from Sanitation Entrepreneurs. Technological Forecasting and Social Change, 79(4), 676-687, 2012.

Reckwitz, A. Toward a Theory of Social Practices: A Development in Culturalist Theorizing. European Journal of Social Theory, 5(2), 243-263, 2002.

Rodrigues, M. A. de S.; Chimenti, P. C. P. de S.; Nogueira, R. R. Adoção de inovações em mercados em rede: uma análise da introdução do livro didático digital no Brasil. Revista de Administração e Inovação, 11(4), 159-192, 2014.

Rodríguez-Barreiro, L. M. et al. Approach to a Causal Model between Attitudes and Environmental Behaviour. A Graduate Case Study. Journal of Cleaner Production, 48, 116-125, 2013.

Rogers, E. M. Diffusion of Innovations. New York: The Free Press, 1995.

Roysen, R. Ecovilas e a construção de uma cultura alternativa. São Paulo, Dissertação (Mestrado em Psicologia Social) - USP, 2013. Disponível em: <www.teses.usp.br/ teses/disponiveis/47/47134/tde-31072013-114650/+\&cd= $1 \& \mathrm{hl}=\mathrm{en} \& \mathrm{ct}=\mathrm{clnk} \& \mathrm{gl}=\mathrm{br}>$.

Roysen, R. Mudança cultural e sustentabilidade: estudo de caso em uma ecovila no Brasil. Anais do $7^{\circ}$ ENANPPAS, 2015. Disponível em: <https://www.researchgate.net/
publication/283710375_Mudanca_cultural_e_sustentabilidade_estudo_de_caso_em_uma_ecovila_no_Brasil>

Salazar, C. A. P. Participación y Acción Colectiva en los Movimientos Globales de Ecoaldeas y Permacultura. Revista Latinoamericana de Psicologia, 45(3), 401-413, 2013.

Santos, P. F. A. A.; Gonçalves, C. A. Difusão de inovações entre organizações: evidências de um estudo na cadeia automobilística. Revista de Administração e Inovação, 12(3), 135-156, 2015.

Schultz, P.W.; Nolan, J. M.; Cialdini, R. B.; Goldstein, N. J.; Griskevicius, V. The Constructive, Destructive, and Reconstructive Power of Social Norms. Psychological Science, 18(5), 429, 2007. Disponível em: <http://hb.summon. serialssolutions.com/link/0/eLvHCXMwY2BQSDYxNU1NNDVLMjI0S0k1Tks0NTZKNkk2SjFKTEtONkpGubkBqTR3E2WQc3MNcfbQhZWK8Sk5OfGgkRJDMyPQlkgxBhZgpzgVAK7zGEs>.

Schwarz, N.; Ernst, A. Agent-Based Modeling of the Diffusion of Environmental Innovations - An Empirical Approach. Technological Forecasting and Social Change, 76(4), 497-511, 2009.

Seyfang, G. Community Action for Sustainable Housing: Building a Low-Carbon Future. Energy Policy, 38(12), 7624-7633, 2010. doi: 10.1016/j.enpol.2009.10.027

Seyfang, G.; Haxeltine, A. Growing Grassroots Innovations: Exploring the Role of Community-Based Initiatives in Governing Sustainable Energy Transitions. Environment and Planning C: Government and Policy, 30(3), 381-400, 2012.

Seyfang, G.; Haxeltine, A.; Hargreaves, T.; Longhurst, N. Energy and Communities in Transition: towards a New Research Agenda on Agency and Civil Society in Sustainability Transitions.Working Paper, 2010. Disponível em: $<$ https://grassrootsinnovations.org/2010/09/21/energy-and-communities-in-transition/>.

Seyfang, G.; Longhurst, N. Desperately Seeking Niches: Grassroots Innovations and Niche Development in the Community Currency Field. Global Environmental Change, 23(5), 881-891, 2013. doi: 10.1016/j.gloenvcha.2013.02.007

Seyfang, G.; Park, J. J.; Smith, A. A Thousand Flowers Blooming? An Examination of Community Energy in the UK. Energy Policy, 61, 977-989, 2013. doi: 10.1016/j. enpol.2013.06.030 
Seyfang, G.; Smith, S. Grassroots Innovations for Sustainable Development: Towards a New Research and Policy Agenda. Environmental Politics, 16(4), 584-603, 2007.

Shove, E.; Pantzar, M.Consumers, Producers and Practices Understanding the Invention and Reinvention of Nordic Walking. Journal of Consumer Culture ARTICLE, 5(1), 43-64, 2005.

Shove, E.; Pantzar, M.; Watson, M. The Dynamics of Social Practice: Everyday Life and How It Changes. London: SAGE Publications Ltd., 2012.

Shove, E.; Walker, G. Governing Transitions in the Sustainability of Everyday Life. Research Policy, 39(4), 471-476, 2010. doi: 10.1016/j.respol.2010.01.019

Shove, E.; Watson, M.; Spurling, N. Conceptualizing Connections: Energy Demand, Infrastructures and Social Practices. European Journal of Social Theory, 18(3), 274287, 2015. doi: 10.1177/1368431015579964

Sidiras, D. Simulation of the Solar Hot Water Systems Diffusion: The Case of Greece. Renewable Energy, 29(6), 907-919, 2004.

Smith, A. Translating Sustainabilities between Green Niches and Socio-Technical Regimes. Technology Analysis \& Strategic Management, 19(4), 427-450, 2007.

Smith, A.; Fressoli, M.; Thomas, H. Grassroots Innovation Movements: Challenges and Contributions. Journal of Cleaner Production, 63, 114-124, 2014. doi: 10.1016/j. jclepro.2012.12.025

Smith, A.; Raven, R. What Is Protective Space? Reconsidering Niches in Transitions to Sustainability. Research Policy, 41(6), 1025-1036, 2012. doi: 10.1016/j.respol.2011.12.012

Smith, A.; Voß, J. P.; Grin, J. Innovation Studies and Sustainability Transitions: The Allure of the Multi-Level
Perspective and Its Challenges. Research Policy, 39(4), 435-448, 2010.

Spaargaren, G. Theories of Practices: Agency, Technology, and Culture. Exploring the Relevance of Practice Theories for the Governance of Sustainable Consumption Practices in the New World-Order. Global Environmental Change, 21(3), 813-822, 2011. doi: 10.1016/j.gloenvcha.2011.03.010

Strengers, Y. Peak Electricity Demand and Social Practice Theories. In: Fudge, S.; Peters, M.; Hoffman, S. M.; Wehrmeyer, W.(Orgs.). The Global Challenge of Encouraging Sustainable Living: opportunities, barriers, policy and practice. Glos: Edward Elgar Publishing Limited, 2013. p. 18-42.

Swilling, M.; Annecke, E. Building Sustainable Neighbourhoods in South Africa: Learning from the Lynedoch Case. Environment and Urbanization, 18(2), 315-332, 2006.

Tanner, C. Constraints on Environmental Behaviour. Journal of Environmental Psychology, 19(2), 145-157, 1999.

Thomas, C.; Sharp, V. Understanding the Normalisation of Recycling Behaviour and Its Implications for Other pro-Environmental Behaviours: A Review of Social Norms and Recycling. Resources, Conservation and Recycling, 79, 11-20, 2013.

Valente, T. W.; Watkins, S. C.;J ato, M. N.; van der Straten, A.; Tsitsol, L. P. M. Social Network Associations with Contraceptive Use among Comeroonian Women in Voluntary Associations. Social Science \& Medicine, 45(5), 677-687, 1997.

Valente, T. W. Social Network Tresholds in the Diffusion of Innovations. Social Network, 18(95), 69-89, 1996.

Veteto, J. R.; Lockyer, J. Environmental Anthropology Engaging Permaculture: Moving Theory and Practice Toward Sustainability. Agriculture, 30(1-2), 47-58, 2008. doi: 10.1111/j.1556-486X.2008.00007.x 